\title{
Kapitalmarktverfassung, Managerentlohnung und Bilanzpolitik
}

von

Günter Franke

\section{Zusammenfassung}

In diesem Beitrag wird untersucht, welchen Einfluss die Entlohnung eines Managers mit Fixum und gewinnbezogenem Bonus auf seinen Arbeitseinsatz und seine Bilanzpolitik ausübt. Dazu wird das LEN-Modell auf die gesamte Lebensdauer des Unternehmens erweitert. Die Gewinnmanipulationen müssen sich über die gesamte Lebensdauer zu null addieren. Es zeigt sich, dass der Manager zu hoher Manipulation tendiert, wenn die Wahrscheinlichkeit, einen Bonus zu erzielen, im Zeitablauf stark schwankt. Durch Anpassung der Basiskurse der Bonuszahlungen an die erwarteten Gewinne kann das Manipulationsverhalten deutlich eingeschränkt werden. Einige Schlussfolgerungen für geeignete Entlohnungssysteme werden abgeleitet. 


\title{
Kapitalmarktverfassung, Managerentlohnung und Bilanzpolitik
}

von

\author{
Günter Franke
}

\section{Kapitalmarktverfassung und Erfolgsmessung}

Man stelle sich einen Wettkampf von Eiskunstläufern vor. Gemäß den Wettkampfregeln bewertet jeder Läufer selbst seine Leistung. Die Wettkampfleitung hat einen Katalog von Regeln verfasst, die bei der Beurteilung zu beachten sind. Außerdem schreiben diese Regeln vor, dass jeder Läufer selbst einen Schiedsrichter einzustellen und zu bezahlen hat, der die Einhaltung der Regeln zu überwachen hat. Schließlich beobachten Zeitungsreporter die Läufer und berichten darüber. In besonders gravierenden Fällen können Läufer, die die Wettkampfregeln missachten, gerichtlich verfolgt werden.

Eine solche Wettkampfordnung erscheint absurd. Niemand käme auf die Idee, den Läufer sich selbst beurteilen zu lassen. Ebenso sollen keinerlei persönliche Beziehungen zwischen Wettkämpfern und Schiedsrichtern bestehen, eine unabdingbare Voraussetzung für eine objektive Kontrolle seitens der Schiedsrichter. Inwieweit Reporter unvoreingenommen berichten und so zu einer objektiven Kontrolle beitragen, ist kontrovers.

Diese seltsame Wettkampfordnung gilt indessen für Kapitalgesellschaften. Die Manager dieser Gesellschaften sind die Hauptakteure bei der Erstellung des Jahresabschlusses, dem wichtigsten Instrument zur Messung des Unternehmenserfolges wie auch des Erfolgs der Manager. Zwar sind sie zahlreichen Regeln der Erfolgsmessung unterworfen, aber diese räumen ihnen erhebliche Ermessensspielräume ein. Sie sind verpflichtet, selbst (mit Zustimmung der Hauptversammlung) Wirtschaftsprüfer anzuwerben und (auf Kosten der Aktionäre) zu bezahlen, die die Erstellung des Jahresabschlusses kontrollieren sollen. Wertpapieranalysten ergänzen die Kontrolle, indem sie die Jahresabschlüsse analysieren und daraus Empfehlungen für Anleger ableiten. Allerdings werden auch die Wertpapieranalysten nicht von den Anlegern bezahlt, sondern von Kreditinstituten und Wertpapierhäusern, die bemüht sind, mit 
Hilfe der Analysen ihrer Mitarbeiter möglichst viele Wertpapiertransaktionen zu generieren. Dies lässt auch die Rolle der Wertpapieranalysten in bezug auf die Kontrolle der Manager in zweifelhaftem Licht erscheinen.

Dass diese seltsame Wettkampfordnung für Manager dem Ziel einer wirksamen Managerkontrolle nicht genügt, hat sich in der jüngsten Zeit in zahlreichen westlichen Ländern gezeigt. Am Neuen Markt in Deutschland hat es zahlreiche Bilanzfälschungen gegeben, die offenbar auch nicht durch potentielle gerichtliche Sanktionen verhindert wurden. In den USA sind ebenfalls zahlreiche Bilanzen gefälscht worden. Diese Fälschungen haben es den Managern solcher Gesellschaften ermöglicht, sich in erheblichem Umfang zu Lasten der Aktionäre $\mathrm{zu}$ bereichern. So wurden durch Schönung von Abschlußzahlen die Aktienkurse nach oben getrieben; dies ermöglichte den Managern, ihre Aktienoptionen mit hohen Gewinnen auszuüben. Die Wirtschaftsprüfer haben es versäumt, Bilanzfälschungen zu verhindern, da sie ihre Honorare nicht gefährden wollten. Wertpapieranalysten haben die Anleger mit falschen Aussagen irregeführt, weil sie dafür von den Managern mit lukrativen Finanzgeschäften belohnt wurden.

Auch wenn es unberechtigt ist, alle Wirtschaftsprüfer und Wertpapieranalysten in einen Topf zu werfen, so muß doch festgestellt werden, dass diese seltsame Wettkampfordnung für Manager den in sie gesetzten Erwartungen nicht gerecht wird. Gemäß diesen Erwartungen vermittelt der Jahresabschluss einen wahren, unverzerrten und informativen Einblick in die Vermögens-, Ertrags- und Finanzlage des Unternehmens. Die Wettkampfordnung ist ein wichtiger Teil der Kapitalmarktverfassung. Für einzelne Elemente dieser Verfassung lassen sich indessen gute Gründe anführen. So macht es Sinn, dass die Manager bei der Erstellung des Jahresabschlusses mitwirken. Denn zahlreiche Komponenten des Periodenerfolgs eines Unternehmens lassen sich nicht anhand ausschließlich vergangenheitsorientierter Daten bemessen, sondern zeigen sich erst in zukünftigen Perioden. Dies gilt insbesondere für Investitionen in Forschung und Entwicklung, aber auch für Ausgaben für Werbung und Organisation und die Bildung von Rückstellungen. Die Abschätzung solcher Zukunftswirkungen setzt Insiderinformationen voraus, die am ehesten den Managern zur Verfügung stehen. Sollen diese Informationen bereits im Periodenerfolg 
berücksichtigt werden, dann kann dies ohne Mitwirkung der Manager kaum praktiziert werden.

Gleichzeitig eröffnet sich aber hiermit ein erheblicher Spielraum zur Ergebnismanipulation oder, neutraler formuliert, zur Bilanzpolitik. Jedes System von Rechnungslegungsvorschriften steht daher im Spannungsfeld von Zukunftsorientierung der Ergebnismessung und Vermeidung von Ergebnismanipulation. Dieses Spannungsfeld hat sich in jüngerer Zeit erheblich ausgeweitet durch den Ausbau der erfolgsabhängigen Managerentlohnung. Da die erfolgsabhängigen Teile der Entlohnung erheblich aufgestockt wurden, sieht der Manager heute seine Bilanzpolitik viel stärker unter dem Aspekt, wie sie auf seine Entlohnung zurückwirkt. Der sinnvollen Idee, Manager durch Kopplung ihrer Entlohnung an den Unternehmenserfolg zu mehr Leistung $\mathrm{zu}$ motivieren, müssen die Fehlanreize zur Ergebnismanipulation gegenübergestellt werden. An dieser Abwägung hat es in der Vergangenheit gemangelt. Zum einen wurde zu viel Vertrauen in die Regeln der Rechnungslegung und die Kontrollmechanismen gesetzt, zum anderen wurde $\mathrm{zu}$ viel Vertrauen in die Informationseffizienz des Kapitalmarktes gesetzt. Beides ist inzwischen einer Ernüchterung gewichen. Es bleibt aber die Frage, wie die sichtbar gewordenen Mängel der Kapitalmarktverfassung eingeschränkt werden können. Dies ist nicht nur unmittelbar für die Verbesserung der Funktionsweise des Kapitalmarkts von Bedeutung, sondern auch mittelbar für die Akzeptanz der Marktwirtschaft.

Zahlreiche Gesetzesinitiativen versuchen, die beobachteten Mängel abzuschwächen. So werden in westlichen Ländern die Beratungsaktivitäten von Wirtschaftsprüfern eingeschränkt, um Interessenkonflikte abzuschwächen. Ebenfalls werden Wertpapieranalysten gezwungen, mögliche Interessenkollisionen $\mathrm{zu}$ offenbaren. Corporate Government Kodizes sollen die Manager zu ehrbarem Verhalten verpflichten (Claussen/Bröcker 2002). Schließlich müssen Chief Executive Officer und Chief Financial Officer gemäß dem im Jahr 2002 verabschiedeten Sarbanes-Oxley-Act die Richtigkeit der von ihnen vorgelegten Zahlen unter Androhung von Strafen und Schadensersatzforderungen bezeugen.

Verschiedentlich wird vermutet, dass die bekannt gewordenen Skandale eher Ausnahmen darstellen. Auch wenn dies vielleicht für Skandale zutrifft, so zeigen jüngere Untersuchungen doch systematische Zusammenhänge zwischen 
Belohnungssystem und Bilanzpolitik. Degeorge/Patel/Zeckhauser [2002] argumentieren, dass Manager danach beurteilt werden, ob die Gewinne der von ihnen geführten Unternehmen bestimmte Schwellenwerte überschreiten. Anhand empirischer Daten belegen sie, dass Bilanzen frisiert werden, um solche Schwellenwerte zu erreichen. Gao/Shrieves [2002] zeigen anhand von Daten, dass ein positiver Zusammenhang zwischen der Anreizintensität von Aktienoptionsprogrammen und der „Gewinnmanagement-Intensität“ besteht. Hierbei spielen Schwellenwerte für Ergebnisse ebenfalls eine Rolle.

$\mathrm{Zu}$ trennen ist die Diskussion um Managerentlohnung und Bilanzpolitik von der Diskussion um Managerentlohnung und Risikopolitik der Manager. Risikopolitik bezeichnet hierbei die Politik, die das Risiko der Realinvestitionen des Unternehmens bestimmt. Bereits 1977 hat Myers gezeigt, dass beschränkt haftende Gesellschafter einen Anreiz haben, riskantere Investitionen zu realisieren, um sich zu Lasten der Unternehmensgläubiger zu bereichern. Gillenkirch [1997, Kap. V] leitet optimale Anreizverträge zur Steuerung des Risikoverhaltens der Manager ab. Er zeigt in Analogie zu Myers, was geschieht, wenn der Manager neben einem Fixum eine Option erhält. Der Manager haftet dann nicht für Unterschreitungen des Ergebnisses, ab dem die optionale Vergütung gezahlt wird. Carpenter [2000] analysiert in einem dynamischen Modell das Risikoverhalten eines Portfolio-Managers, der neben einem Fixum eine Option auf seinen Anlageerfolg bekommt. Der Manager wählt eine Politik, die einen Gewinn hoch über dem Basiskurs der Option oder weit darunter erzeugt. Jedoch muss die Option das Risiko nicht in jeder Situation erhöhen. Das/Sundaran [2002] ergänzen eine ähnliche Analyse um Signalisierungsaspekte und zeigen, dass eine Entlohnung unter Einschluss von Optionen das Wohl der Anleger auch erhöhen kann.

Im folgenden Beitrag soll der Zusammenhang zwischen der erfolgsabhängigen Entlohnung der Manager und ihrer Bilanzpolitik untersucht werden. Hierbei soll ein sehr einfaches Modell verwendet werden, aufbauend auf dem bekannten LEN-Modell (Spremann 1987). Unterstellt wird eine gewinnabhängige Entlohnung mit Optionscharakter. Wenn der Gewinn eine vorgegebene Höhe überschreitet, erhält der Manager einen Anteil des darüber hinaus gehenden Gewinns. Gleichzeitig weiß der Manager, dass eine Gewinnerhöhung in einer Periode eine gleich hohe Gewinnminderung in zukünftigen Perioden nach sich zieht, soweit diese 
Gewinnänderungen ausschließlich Ergebnis der Bilanzpolitik sind. Der Manager rechnet mit Sanktionen für Gewinnmanipulationen, die umso höher sind, je höher die Manipulationen sind. Dieses Umfeld kann für den Manager einen Anreiz erzeugen, den Gewinn in einzelnen Perioden $\mathrm{zu}$ erhöhen und dementsprechend in anderen $\mathrm{zu}$ vermindern. Entscheidend für dieses Ergebnis ist die mit einer optionsähnlichen Entlohnung implizit verbundene Haftungsbeschränkung. Sie erlaubt dem Manager, sich zu Lasten der Aktionäre zu bereichern. Die Aktionäre sollten daher versuchen, eine erfolgsabhängige Entlohnung zu konzipieren, die einer solchen Bereicherung möglichst entgegen wirkt.

Im folgenden Abschnitt 2 wird das Entscheidungsmodell des Managers vorgestellt und seine optimale Politik abgeleitet. Im Abschnitt 3 werden mögliche Konsequenzen für die Gestaltung der Entlohnungspolitik diskutiert. Im vierten Abschnitt werden die Implikationen für Aktienoptionspläne und Aktienpläne erörtert, ein Fazit wird am Schluß gezogen.

\section{Ein einfaches Entscheidungsmodell des Managerverhaltens}

Im Rahmen der Agency-Theorie ist eine Fülle von Modellen zur optimalen Gestaltung von erfolgsabhängigen Entlohnungssysstemen entwickelt worden (Laux 2003, Teil F; Murphy 1999). Unterstellt wird hierbei im allgemeinen, dass der Manager bereit ist, seinen Arbeitseinsatz zu erhöhen, wenn er dafür belohnt wird. Da sein Arbeitseinsatz nicht beobachtbar ist, wohl aber der dadurch beeinflusste Unternehmenserfolg, kann die Entlohnung nur an diesen anknüpfen, nicht aber an den Arbeitseinsatz selbst. Auf den Unternehmenserfolg wirken auch andere Faktoren ein, so dass der Unternehmenserfolg nur einen probabilistischen Rückschluss auf den Arbeitseinsatz zulässt. Grundsätzlich erweist sich eine Erfolgsbeteiligung als vorteilhaft für die Prinzipale.

Diese Modelle unterstellen, dass der Erfolg eindeutig messbar ist, insbesondere dass der Erfolgsausweis nicht vom Manager manipuliert werden kann (siehe hierzu auch Laux 1999, Kap. VI, VII). Gerade hier setzt dieser Beitrag an. Ausgangspunkt ist die Annahme, dass der Unternehmenserfolg am Gewinn gemessen wird. Da der Manager Bilanzpolitik betreiben kann, kann er Gewinne zwischen den Perioden der Rechnungslegung verschieben. Zugrunde liegt ein Rechnungslegungsrecht, wonach 
über die gesamte Lebensdauer des Unternehmens der gesamte Einzahlungsüberschuss des Unternehmens übereinstimmt mit der Summe der Gewinne, korrigiert um Kapitaleinzahlungen und -rückzahlungen sowie Gewinnentnahmen. Im deutschen Recht, aber auch in zahlreichen anderen Rechtsordnungen ist diese Bedingung im wesentlichen gesichert. Bei gegebenen Zahlungsströmen des Unternehmens beschränkt sich die Bilanzpolitik dann auf die zeitliche Umverteilung der Gewinne über die verschiedenen Perioden der Rechnungslegung.

Um das Entscheidungsmodell einfach zu halten, gehen wir vom LEN-Modell aus. Dieses Modell ist insbesondere durch drei Annahmen gekennzeichnet. 1. Der Manager erhält neben einem Fixum einen konstanten Anteil des Erfolgs als Entlohnung. 2. Die Risikopräferenz des Managers ist durch konstante absolute Risikoaversion gekennzeichnet, er besitzt also eine exponentielle Nutzenfunktion. 3. Der ohne Manipulation ermittelte Erfolg gehorcht einer Normalverteilung, wobei die Standardabweichung des Erfolgs exogen vorgegeben ist, also nicht von der Politik des Managers abhängt. Mit dieser Annahme wird das bekannte Thema von Rückwirkungen des Agenten auf das Erfolgsrisiko ausgeklammert (Myers 1977, Franke/Hax 1999, S. 421 ff, Laux 1999, Kap. XIII). Damit wird auch eine indirekte Rückwirkung der Entlohnungspolitik auf das Erfolgsrisiko ausgeklammert. (Carpenter 2000, Gillenkirch 1997, Kap. V). Diese Annahme wird in diesem Beitrag beibehalten.

Das LEN-Modell wird hier um verschiedene Aspekte erweitert.

a) Es wird die gesamte Lebensdauer des Unternehmens betrachtet. Diese liege mit $T$ Perioden fest. Intertemporale Aspekte werden hier weitgehend ausgeklammert. So wirkt der Arbeitseinsatz des Managers in Periode $t$ nur auf den Gewinn derselben Periode. Lediglich Gewinnmanipulationen führen $\mathrm{zu}$ Verschiebungen von Gewinnen über Perioden. Die exogenen zufälligen Gewinnstörungen in den einzelnen Perioden seien voneinander unabhängig.

b) Der Manager entscheidet in einer Periode über Arbeitseinsatz und Gewinnmanipulation, bevor die Höhe der Gewinnstörung in dieser Periode bekannt ist. In der letzten Periode entfällt die Entscheidung über die Gewinnmanipulation, da diese bereits durch die diesbezüglichen vorangehenden Entscheidungen festliegt. 
Diese Annahme ist nicht sehr realistisch, sie dient der Vereinfachung des Modells. Sie wirkt weniger problematisch bei kurzen Perioden, da die Bilanzpolitik kurzfristig nicht massiv geändert werden kann.

c) Der Manager erhält neben dem Fixum einen Bruchteil des ausgewiesenen Gewinns, soweit dieser einen vorgegebenen Mindesterfolg $B$ übersteigt. Die erfolgsabhängige Entlohnung hat daher dasselbe Auszahlungsprofil wie eine Kaufoption auf den Gewinn mit dem Basiskurs $B$. Hiermit wird eine Haftungsbeschränkung für den Manager unterstellt; für Verluste und für Gewinne unter dem Basiskurs $B$ wird er mit seinem Gehalt nicht zur Verantwortung gezogen.

d) Die Zahlung einer erfolgsabhängigen Entlohnung vermindert die risikofreie Geldanlage des Unternehmens. Der Habenzinssatz ist gleich 0.

e) Gewinnmanipulationen bedeuten nicht nur eine zeitliche Verschiebung von Gewinnen, sondern erzeugen Kosten für den Manager. Die direkten Kosten reflektieren potentielle Nachteile des Managers, die bei Aufdeckung der Manipulationen entstehen. Solche Nachteile schließen einen Vertrauensverlust bei den Aktionären ein ebenso wie bei gravierenden Manipulationen die Gefahr gerichtlicher Maßnahmen. Die direkten Kosten einer Gewinnmanipulation wachsen quadratisch mit der Höhe der Manipulation. Auch hier wird der Einfachheit halber unterstellt, dass die Kosten nur in den Manipulationsperioden selbst anfallen. Da eine Gewinnverschiebung jedoch mindestens zwei Perioden berührt, fallen Kosten in mindestens zwei Perioden an. Indirekte Kosten der Gewinnmanipulation entstehen dadurch, dass eine Gewinnerhöhung in einer Periode zwangsläufig zu einer Gewinnminderung in anderen Perioden führt. Das Einkommen des Managers wird folglich in diesen Perioden tendenziell reduziert. Die Position des Managers wird nicht gefährdet, er bleibt bis zur Liquidation des Unternehmens im Amt. 
Folgende Symbole werden verwendet:

$k_{t} \quad$ erwarteter Gewinn in Periode $t$ bei minimalem Arbeitseinsatz des Managers vor Gewinnmanipulation und vor Abzug der Vergütung des Managers;

$e_{t} \quad$ über den minimalen Arbeitseinsatz hinausgehender Arbeitseinsatz des Managers in Periode $t ; e_{t} \geq 0$; der Gewinn wächst infolge dieses Arbeitseinsatzes um $e_{t}$;

$\Delta_{t} \quad$ Veränderung des Gewinnausweises in Periode $t$ durch Manipulation; $\Delta_{t} \underset{<}{\stackrel{>}{=} 0}$

$y_{t} \quad$ exogene, zufällige Gewinnstörung in Periode $t$; sie ist normalverteilt mit Erwartungswert 0 und Standardabweichung $\sigma_{t}$;

$B_{t} \quad$ Gewinnschwelle in Periode $t$, ab der eine erfolgsabhängige Vergütung gezahlt wird (Basiskurs);

$\gamma_{t}$ Beteiligungsquote des Managers an Gewinn abzüglich Basiskurs in Periode $t$;

$c_{e}, c_{\Delta}$ Kostenkonstanten;

b absolute Risikoaversion des Managers;

$f_{t} \quad$ Fixum des Managers in Periode $t$

$\mu_{t} \quad$ erwarteter Gewinnausweis in Periode $t$, nach Abzug des Fixums, vor Abzug der gewinnabhängigen Vergütung

$\mu_{t}=k_{t}-f_{t}+e_{t}+\Delta_{t}$

$g_{t}$ zufallsabhängiger Gewinnausweis des Unternehmens in Periode $t$ $g_{t}=\mu_{t}+y_{t}$

$\beta_{t} \quad$ Zeitpräferenzfaktor des Managers; $\beta_{t} \in(0,1) ; \beta_{t}>\beta_{t+1}$; 
Das gesamte Entgelt des Managers in Periode $t$ beträgt

$f_{t}+\gamma_{t} \max \left\{0, g_{t}-B_{t}\right\}$

Der Manager hat eine zeitadditive Nutzenfunktion, die separabel ist in seinem Entgelt und den Kosten des Arbeitseinsatzes sowie den Kosten der Gewinnmanipulation. Sein erwarteter Nutzen in Periode $t$ ist

$U_{t}=E\left[u\left(f_{t}+\gamma_{t} \max \left\{0, g_{t}-B_{t}\right\}\right)\right]-c_{e} e_{t}^{2}-c_{\Delta} \Delta_{t}^{2}$

$c_{e}$ determiniert somit das marginale Arbeitsleid $2 c_{e} e_{t}$, das erforderlich ist, um den Gewinn um eine weitere Einheit zu erhöhen. Entsprechend determiniert $c_{\Delta}$ die marginalen Kosten $2 c_{e} \Delta_{t}$ einer Gewinnmanipulation. Bei exponentieller Nutzenfunktion folgt

$U_{t}=-E\left[\exp \left(-b\left(f_{t}+\gamma_{t} \max \left\{0, g_{t}-B_{t}\right\}\right)\right)\right]-c_{e} e_{t}^{2}-c_{\Delta} \Delta_{t}^{2}$

Der erste Summand in (1) wächst c.p. mit b. Für hohes b strebt er gegen 0. Die Empfindlichkeit des ersten Ausdrucks gegenüber Änderungen in der Entlohnung ist folglich um so kleiner, je größer b ist. Dementsprechend sollte auch die Empfindlichkeit von $U_{t}$ gegenüber Änderungen des Arbeitseinsatzes oder der Manipulation abnehmen, wenn b größer wird. Folglich sollten $c_{e}$ und $c_{\Delta}$ um so kleiner sein, je höher b ist.

Der Manager maximiert

$\sum_{t=1}^{T} \beta_{t} U_{t}$ unter der Nebenbedingung $\sum_{t=1}^{T} \Delta_{t}=0$

Die Nebenbedingung besagt, dass sich die Gewinnmanipulationen über die gesamte Lebensdauer gegenseitig aufheben müssen. $e_{t}$ und $\Delta_{t} \quad(t=1, \ldots, T)$ sind die Entscheidungsvariablen des Managers. 


\section{Die optimale Bilanzmanipulation}

\subsection{Die Optimumbedingungen}

Bei dem Optimierungsproblem handelt es sich um ein mehrperiodiges Entscheidungsproblem. Im allgemeinen ist die optimale Entscheidung stochastischsequentiell. D.h., die optimale Entscheidung in Periode $t$ hängt von den Entscheidungen der vorangehenden und der nachfolgenden Perioden ab sowie von den Realisationen der Gewinnstörungen $y$ in den vorhergehenden Perioden. Das hier vorgestellte Modell vereinfacht die Zusammenhänge erheblich durch die Annahme, dass die Gewinnstörungen voneinander unabhängig sind und jeweils nur in einer Periode wirken. $\mathrm{Da}$ außerdem die Entscheidungen in einer Periode vor Bekanntwerden der Realisation der Gewinnstörung getroffen werden, sind die optimalen Entscheidungen zustandsunabhängig, d.h. sie sind unabhängig von den Realisationen der Gewinnstörungen der vorhergehenden Perioden und der Gewinnstörung derselben Periode. Der Verbund zwischen den Entscheidungen verschiedener Perioden resultiert ausschließlich aus der Nebenbedingung, wonach sich die (zustandsunabhängigen) Gewinnmanipulationen zu 0 addieren.

Zum Verständnis des Modells wird zunächst der erwartete Nutzen des Managers erläutert. Gleichung (1) lässt sich schreiben als

$$
\begin{gathered}
U_{t}=-\exp \left(-b f_{t}\right)\left[\int_{-\infty}^{B_{t}} d N\left(g_{t} ; \mu_{t}, \sigma_{t}\right)+\int_{B_{t}}^{\infty} \exp \left(-b \gamma_{t}\left(g_{t}-B_{t}\right)\right) d N\left(g_{t} ; \mu_{t}, \sigma_{t}\right)\right] \\
-c_{e} e_{t}^{2}-c_{\Delta} \Delta_{t}^{2}
\end{gathered}
$$

wobei $N\left(g_{t} ; \mu_{t}, \sigma_{t}\right)$ die kumulative Normalverteilung mit den Parametern $\mu_{t}$ und $\sigma_{t}$ bezeichnet. Differenziert man $U_{t}$ nach $\mu_{t}$, so erhält man

$$
\begin{aligned}
\partial U_{t} / \partial \mu_{t} & =b \gamma_{t} \exp \left(-b f_{t}\right) \int_{B_{t}}^{\infty} \exp \left(-b \gamma_{t}\left(g_{t}-B_{t}\right)\right) d N\left(g_{t} ; \mu_{t}, \sigma_{t}\right) \\
& =b \gamma_{t} \exp \left[-b\left(f_{t}+\frac{1}{2} b\left(\gamma_{t} \sigma_{t}\right)^{2}\right)\right] \exp \left(-b \gamma_{t} \sigma_{t} v_{t}\right) N\left(v_{t}\right)
\end{aligned}
$$


wobei

$v_{t}=\left(\mu_{t}-B_{t}-b \gamma_{t} \sigma_{t}^{2}\right) / \sigma_{t}$

und $N\left(v_{t}\right)$ der Wert der standardisierten Normalverteilung an der Stelle $v_{t}$ ist. Gleichung (3) wird im Anhang abgeleitet.

Zum Verständnis von Gleichung (3) ist es zweckmäßig, sie umzuschreiben zu

$\partial U_{t} / \partial \mu_{t}=b \gamma_{t} \exp \left[-b\left(f_{t}+\gamma_{t}\left(\mu_{t}-B_{t}\right)-\frac{1}{2} b\left(\gamma_{t}^{2} \sigma_{t}^{2}\right)\right)\right] N\left(v_{t}\right)$

$f_{t}+\gamma_{t}\left(\mu_{t}-B_{t}\right)$ ist die erwartete Entlohnung des Managers, wenn er auf jeden Fall eine Erfolgsbeteiligung erhält. $1 / 2 b\left(\gamma_{t}^{2} \sigma_{t}^{2}\right)$ ist die Risikoprämie, die der Manager bei exponentieller Nutzenfunktion für das Risiko $\gamma_{t} \sigma_{t}$, das er zu tragen hat, geltend macht. $f_{t}+\gamma_{t}\left(\mu_{t}-B_{t}\right)-1 / 2 b\left(\gamma_{t}^{2} \sigma_{t}^{2}\right)$ ist also das erwartete Einkommen des Managers unter Abzug der Risikoprämie, ausgehend davon, dass er auf jeden Fall eine Gewinnbeteiligung erhält. Diese Prämisse trifft jedoch, anders als im klassischen LENModell, hier nicht zu. Daher ist der Exponentialausdruck in Gleichung (5) mit $N\left(v_{t}\right) \mathrm{zu}$ multiplizieren. $N\left(v_{t}\right)$ ist die risikoadjustierte Erfolgswahrscheinlichkeit, also die Wahrscheinlichkeit, dass der Manager eine Gewinnbeteiligung erhält. Berechnet wird diese Wahrscheinlichkeit anhand der „wahren“ Wahrscheinlichkeitsdichte, multipliziert mit dem auf 1 standardisierten Grenznutzen des Einkommens des Managers (siehe Anhang). Der ,, Grenzertrag “ $\partial U_{t} / \partial \mu_{t}$ ist also gleich dem Grenznutzen einer Zunahme des erwarteten Managereinkommens, multipliziert mit der Erfolgswahrscheinlichkeit.

Der Grenzertrag hängt von der Beteiligungsquote und der Erfolgswahrscheinlichkeit in der betreffenden Periode ab. Eine Erhöhung der Beteiligungsquote bewirkt wie im klassischen LEN-Modell eine Erhöhung der erwarteten Entlohnung, aber auch ein höheres vom Manager zu tragendes Risiko, da er einen höheren Anteil der Gewinnstörungen übernimmt. Der Nachteil daraus zeigt sich in der Risikoprämie $1 / 2 b \gamma_{t}^{2} \sigma_{t}^{2}$ 
Da $\partial \mu_{t} / \partial e_{t}=\partial \mu_{t} / \partial \Delta_{t}=1$ ist, lassen sich die notwendigen Bedingungen für ein optimales Managerverhalten leicht angeben: ${ }^{1}$

$e_{t}: \quad \partial U_{t} / \partial \mu_{t}=2 c_{e} e_{t}^{*}, \quad \forall t$

$\Delta_{t}: \quad \partial U_{t} / \partial \mu_{t}=2 c_{\Delta} \Delta_{t}^{*}+\frac{\lambda}{\beta_{t}}, \quad \forall t ;$

wobei $\lambda$ den Lagrange-Multiplikator der Nebenbedingung $\sum_{t=1}^{T} \Delta_{t}=0$ bezeichnet.

Aus den Bedingungen (6) und (7) folgt

$e_{t}^{*}=\left[c_{\Delta} \Delta_{t}^{*}+\frac{\lambda}{2 \beta_{t}}\right] / c_{e}$

Der optimale Arbeitseinsatz ist also eine lineare Funktion der optimalen Gewinnmanipulation. Daraus folgt für den erwarteten Gewinnausweis

$$
\begin{aligned}
\mu_{t} & =k_{t}-f_{t}+e_{t}^{*}+\Delta_{t}^{*} \\
& =k_{t}-f_{t}+\left(1+\frac{c_{\Delta}}{c_{e}}\right) \Delta_{t}^{*}+\frac{\lambda}{2 \beta_{t} c_{e}}
\end{aligned}
$$

Setzt man dies in Gleichung (4) ein, so folgt

$$
v_{t}=\left[k_{t}-f_{t}+\left(1+\frac{c_{\Delta}}{c_{e}}\right) \Delta_{t}^{*}+\frac{\lambda}{2 \beta_{t} c_{e}}-B_{t}-b \gamma_{t} \sigma_{t}^{2}\right] / \sigma_{t}
$$

Damit erweist sich $v_{t}$ als lineare Funktion der optimalen Gewinnmanipulation $\Delta_{t}^{*}$ und des Lagrange-Multiplikators $\lambda$.

Zum Veständnis der Bedingungen für ein Optimum ist es hilfreich, von Gleichung (3) auszugehen. Definiert man den exogenen Parameter

$$
A_{t} \equiv b \gamma_{t} \exp \left[-b\left(f_{t}+\frac{1}{2} b\left(\gamma_{t} \sigma_{t}\right)^{2}\right)\right]
$$

dann folgt für den Grenzertrag

$$
\partial U_{t} / \partial \mu_{t}=A_{t} \exp \left(-b \gamma_{t} \sigma_{t} v_{t}\right) N\left(v_{t}\right)
$$

Da der Grenzertrag stets positiv ist, ergibt sich stets ein positiver optimaler Arbeitseinsatz. Eine Nichtnegativitätsbedingung für den Arbeitseinsatz erübrigt sich daher. 
Der Grenzertrag ist also lediglich eine Funktion von $v_{t}$, das durch $\Delta_{t}^{*}$ und $\lambda$ bestimmt wird. Löst man Gleichung (10) nach $\Delta_{t}^{*}$ auf, so kann man die Bedingung (7) für ein Optimum schreiben als

$\frac{\partial U_{t}}{\partial \mu_{t}}\left(v_{t}\right)=\left(1+\frac{c_{\Delta}}{c_{e}}\right)^{-1}\left[2 c_{\Delta}\left(v_{t} \sigma_{t}+b \gamma_{t} \sigma_{t}^{2}-k_{t}+f_{t}+B_{t}\right)+\frac{\lambda}{\beta_{t}}\right]$

Damit ist diese Bedingung auf $v_{t}$ und $\lambda$ zurückgeführt.

Um die optimale Lösung zu verstehen, ist es zweckmäßig, die beiden Seiten von Gleichung (13) grafisch $\mathrm{zu}$ veranschaulichen. Abbildung 1 veranschaulicht den Grenzertrag, Abbildung 2 das Optimum. 


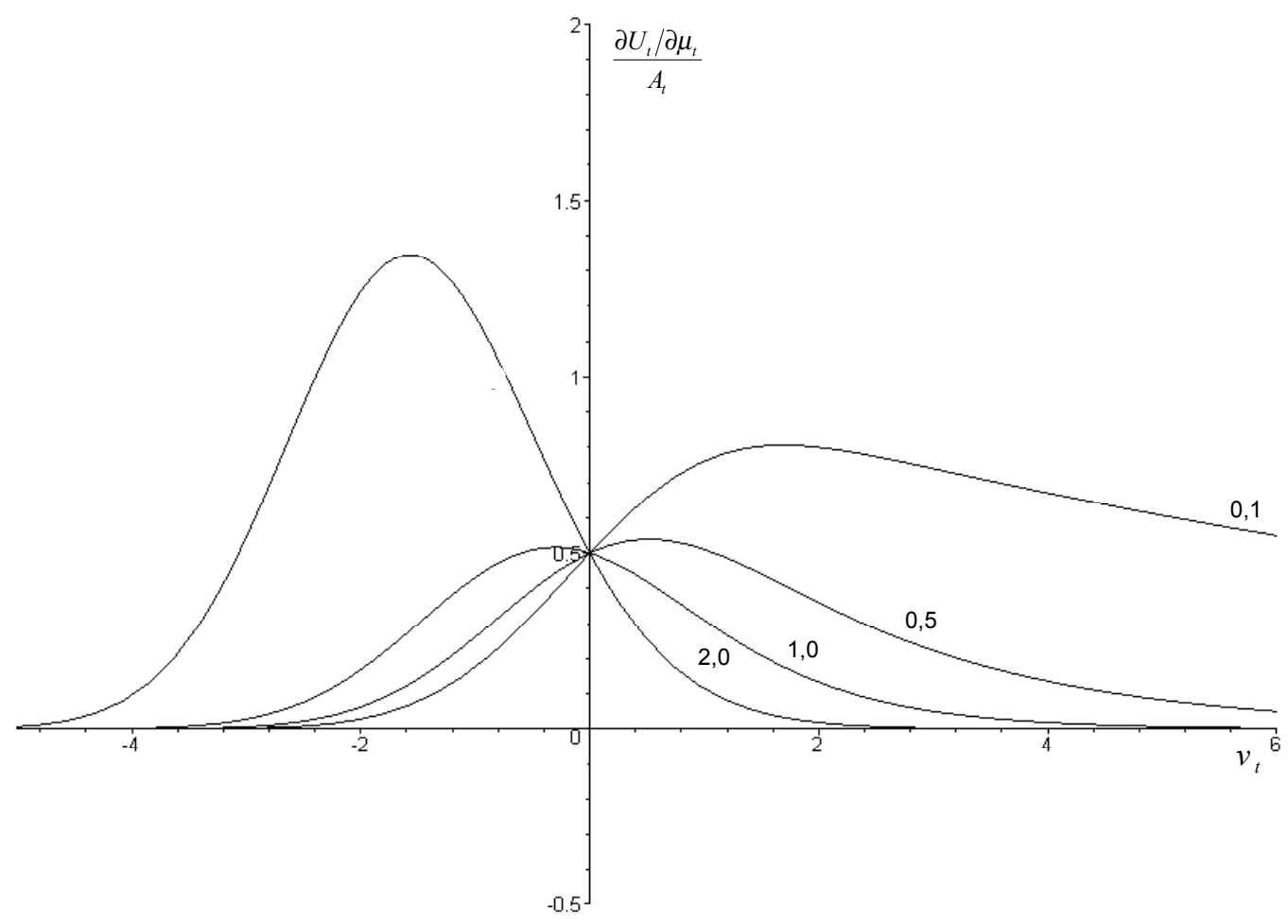

Abb. 1: Die Funktion $\exp \left(-b \gamma_{t} \sigma_{t} v_{t}\right) N\left(v_{t}\right)$ wird für verschiedene Werte von $b \gamma_{t} \sigma_{t}$ gezeigt. Die Werte sind 0,$1 ; 0,5 ; 1,0$ und 2,0.

Wie Abb.1 zeigt, ist die Kurve $\left[\partial U_{t} / \partial \mu_{t}\right] A_{t}^{-1}$ eine unimodulare Kurve, die für $v \rightarrow \pm \infty$ gegen 0 strebt. Jede Kurve weist für $v=0$ den Wert $N(0)=0,5$ auf. Das Maximum der Kurve liegt bei einem $v$-Wert unter [bei] [über] 0 , wenn $b \gamma_{t} \sigma_{t}>[=][<] 0,78=n(0) / N(0) \quad$ ist. $n($.$) bezeichnet die Dichte der$ Standardnormalverteilung. 


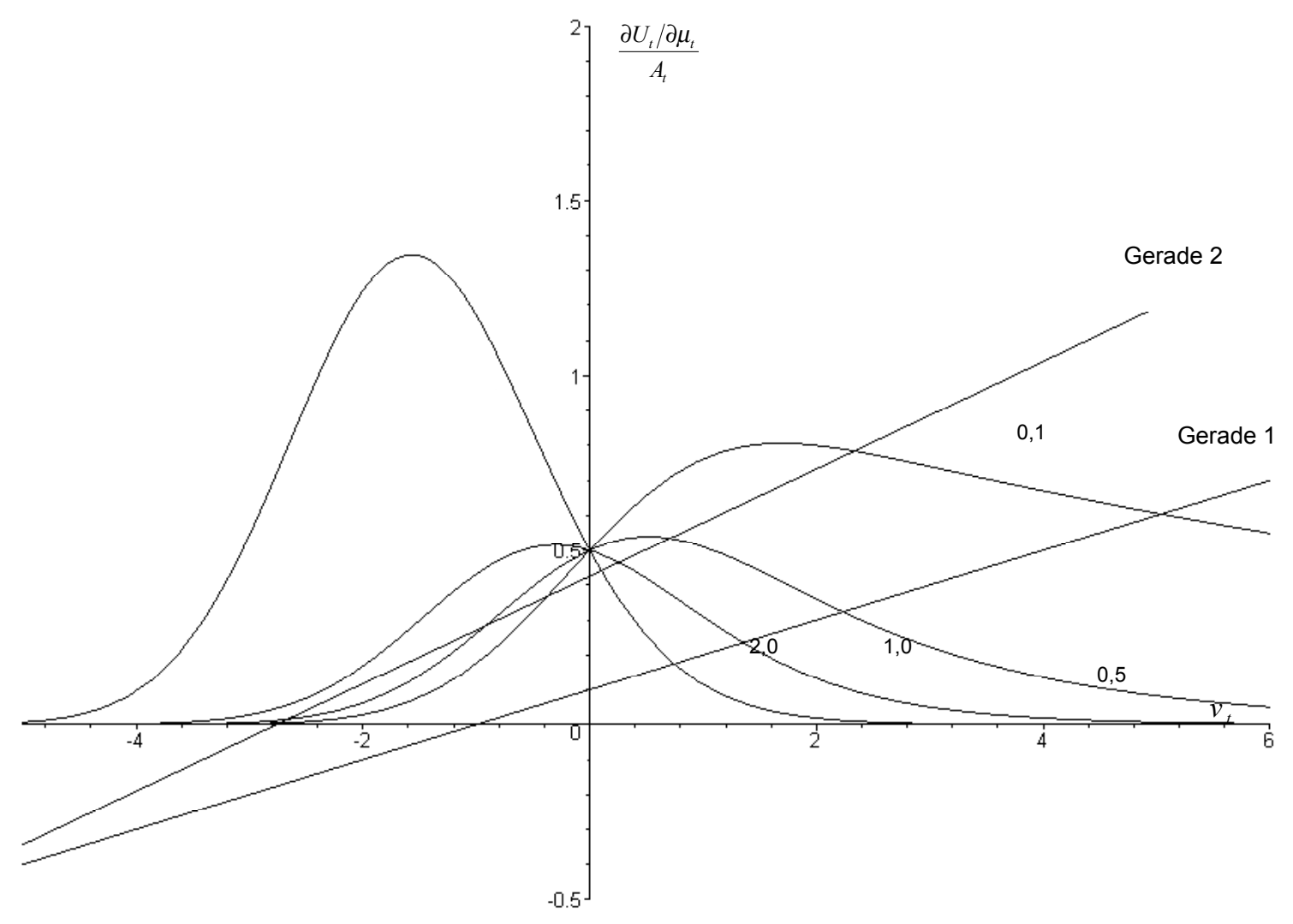

Abb.2: Die Abbildung zeigt dieselben Kurven wie Abb.1 und zusätzlich zwei alternative Geraden, die die rechte Seite von Gleichung (13), dividiert durch $A_{t}$, angeben. Gerade 1 schneidet die gezeigten $\left(\partial U_{t} / \partial \mu_{t}\right) A_{t}^{-1}$-Kurven nur einmal, Gerade 2 schneidet die 0,1- und die 0,5-Kurve dreimal, die 1,0- und die 2,0-Kurve einmal.

Abb. 2 verdeutlicht die Optimumbedingung (13). Die beiden Geraden kennzeichnen jeweils die rechte Seite von Gleichung (13), dividiert durch $A_{t}$. Sie gelten für unterschiedliche Datenkränze. Im Optimum muss der Grenzertrag einer Gewinnmanipulation in Periode $t$ mit den Grenzkosten übereinstimmen. Der Grenzertrag wird durch die linke Seite von Gleichung (13) angegeben, die Grenzkosten durch die rechte Seite. Abb.2 veranschaulicht Grenzertrag und Grenzkosten, dividiert durch $A_{t}$. In einem Schnittpunkt von Kurve und Gerade gilt folglich „Grenzertrag = Grenzkosten“. Gibt es nur einen Schnittpunkt, dann ist die optimale Politik in Periode $t$ durch diesen Schnittpunkt eindeutig bestimmt. Abb.2 zeigt indessen, dass es auch drei 
Schnittpunkte geben $\mathrm{kann}^{2}$. In diesem Fall gibt es ein lokales Maximum beim ersten Schnittpunkt und ein weiteres beim dritten Schnittpunkt. Der mittlere Schnittpunkt bezeichnet ein lokales Minimum und ist daher für den Manager uninteressant.

\subsection{Diskussion der Modelllösung}

Im folgenden wird die Lösung des Modells diskutiert. Gleichung (8) zeigt den Zusammenhang zwischen optimalem Arbeitseinsatz und optimaler Gewinnmanipulation in einer Periode. Bei gegebenem $\lambda$ wächst der optimale Arbeitseinsatz linear mit der optimalen Gewinnmanipulation. Die Erklärung liegt darin, dass sich ein höherer Gewinnausweis auf die Entlohnung der Periode genauso wie eine Senkung des Basiskurses auswirkt und dadurch der Manager mit höherer Wahrscheinlichkeit eine Gewinnbeteiligung erhält. Dies stärkt seinen Leistungsanreiz. Durch die Gewinnmanipulation beeinflußt der Manager auch seinen Arbeitseinsatz. Ebenso erhöht ein höherer Arbeitseinsatz den Gewinnausweis. Denn die Gewinnerhöhung infolge höheren Arbeitseinsatzes wirkt ebenfalls wie eine Senkung des Basiskurses, so dass der Manager mit höherer Wahrscheinlichkeit von einer positiven Gewinnmanipulation profitiert. Hoher (geringer) Arbeitseinsatz und hohe positive (negative) Gewinnmanipulation gehen also Hand in Hand. Hohe Volatilität des Arbeitseinsatzes geht also Hand in Hand mit hoher Volatilität der Gewinnmanipulation, es kann somit zu hoher Verhaltensvolatilität kommen. Es überrascht nicht, dass der optimale Arbeitseinsatz umso höher ist, je geringer das Arbeitsleid, ausgedrückt durch $c_{e}$, ist. Ebenso ist das Ausmaß der Gewinnmanipulation umso größer, je geringer deren direkte Kosten, ausgedrückt durch $c_{\Delta}$, sind.

Im folgenden konzentrieren wir uns auf die Politik der Gewinnmanipulation. Addiert man Gleichung (7) über alle Perioden, so folgt

$$
\lambda=\frac{\sum_{t=1}^{T} \partial U_{t} / \partial \mu_{t}}{\sum_{t=1}^{T} \beta_{t}^{-1}} .
$$

2 Möglich ist auch, dass es einen Schnittpunkt und einen Tangentialpunkt gibt. Im Schnittpunkt liegt ein Maximum vor, im Tangentialpunkt ein Sattelpunkt. 
$\lambda$, die indirekten Kosten einer marginalen Gewinnmanipulation, gleichen also der Summe der Grenzerträge über alle Perioden, dividiert durch die Summe der inversen Zeitpräferenzfaktoren. Damit lässt sich Gleichung (7) umschreiben zu

$$
\Delta_{t}^{*}=\frac{\partial U_{t} / \partial \mu_{t}-\left(\beta_{t}^{-1} / \sum_{\tau} \beta_{\tau}^{-1}\right) \sum_{\tau} \partial U_{\tau} / \partial \mu_{\tau}}{2 c_{\Delta}} .
$$

Bei optimaler Politik ist also die Gewinnmanipulation in einer Periode negativ (positiv), wenn der Grenzertrag dieser Periode kleiner (größer) ist als der gewogene durchschnittliche Grenzertrag. Ob diese Politik zu einer Gewinnglättung oder destabilisierung führt, kann indessen nicht ohne weitere Bedingungen geklärt werden. Dazu betrachten wir verschiedene Situationen.

In der ersten Situation sei der Manager risikoneutral, die direkten Kosten der Gewinnmanipulation seien gleich 0 , also $c_{\Delta}=0$. Der Grenzertrag in Periode $\mathrm{t}$ ist dann gleich $\gamma_{t} N\left(v_{t}\right)$, wobei $v_{t}$ gemäß Gleichung (4) mit $b=0$ berechnet wird. Der Grenzertrag einer Gewinnmanipulation wächst also bei Risikoneutralität monoton mit $v_{t}$. Ist $c_{\Delta}=0$, dann gibt es nur konstante indirekte Grenzkosten der Gewinnmanipulation in Höhe des „durchschnittlichen“ Grenzertrags. Folglich existiert kein inneres Optimum. Der Manager betreibt eine extreme Gewinndestabilisierung. Dies überrascht nicht. Denn infolge des Optionscharakters der Erfolgsbeteiligung ist der Wert der Gewinnbeteiligung umso höher, je stärker die Gewinne im Zeitablauf schwanken. ${ }^{3}$

In der zweiten Situation zeige der Manager konstante absolute Risikoaversion, jedoch seien die direkten Kosten der Gewinnmanipulation wieder gleich 0, also $c_{\Delta}=0$. Folglich verursacht die Gewinnmanipulation nur konstante indirekte Grenzkosten. Die Grenzkostenkurve in Abb. 2 verläuft also wieder horizontal. Hier sind zwei Unterfälle zu unterscheiden.

3 Dieses Resultat ähnelt der Politik eines Portfolio-Managers, der einen optionsähnlichen Erfolgsbonus erhält (Carpenter 2000). 
Unterfall a: Bei sehr geringer Risikoscheu kann es für den Manager optimal sein, die Gewinne in extremer Weise zu manipulieren. Dann konvergiert der Grenzertrag in jeder Periode gegen 0. Extreme Gewinndestabilisierung ist die Folge.

Unterfall b: Mit zunehmender Risikoscheu wird es für den Manager attraktiver, sein Einkommen über die Zeit $\mathrm{zu}$ stabilisieren. Das Instrument hierzu ist die Gewinnmanipulation; die Alternative hierzu wäre eine Politik der Geldaufnahme und -anlage am Kapitalmarkt. Da die Grenzertragskurve unimodal ist, gibt es bei horizontaler Grenzkostenkurve zwei Schnittpunkte von Grenzertrags- und Grenzkostenkurve. Der erste Schnittpunkt markiert ein Minimum, der zweite ein Maximum. Folglich ist die Lösung eindeutig durch den zweiten Schnittpunkt bestimmt. Gemäß Gleichung (7) ist der Grenzertrag, multipliziert mit $\beta$, im Optimum in allen Perioden gleich hoch. Folglich ist auch der optimale Arbeitseinsatz, multipliziert mit $\beta$, in allen Perioden gleich hoch. Wären also die exogenen Daten einschließlich $\beta_{t}$ in allen Perioden gleich hoch, dann gäbe es keine Gewinnmanipulation. Soweit die Daten im Zeitablauf variieren, kommt es zur Gewinnmanipulation. Diese hat allerdings ausschließlich den Zweck, den mit $\beta$ multiplizierten Grenzertrag über alle Perioden gleich hoch $\mathrm{zu}$ gestalten. Die Gewinnmanipulation dient hier also der totalen Verstetigung des Grenzertrags, nicht der totalen Gewinnglättung.

In der dritten Situation zeige der Manager konstante absolute Risikoaversion, die direkten Kosten der Gewinnmanipulation seien positiv, also $c_{\Delta}>0$. Zunächst unterstellen wir, dass $c_{\Delta}$ sehr groß ist. Demnach verursacht eine Gewinnmanipulation sehr hohe Kosten. Dann hat die Grenzkostengerade in Abb. 2 eine sehr hohe Steigung. Es gibt dann nur einen Schnittpunkt zwischen Grenzertrags- und Grenzkostenkurve. Das Optimum ist eindeutig bestimmt. Weil $c_{\Delta}$ sehr groß ist, ist gemäß Gleichung (7) $\Delta_{t}^{*}$ nahe bei 0 . Es kommt zur Gewinnmanipulation, jedoch ist diese in ihrem Ausmaß sehr beschränkt.

Die eigentlich interessante Situation ist die vierte Situation. Sie gleicht der dritten, jedoch ist das Niveau von $c_{\Delta}$ so, dass zumindest in einzelnen Perioden drei Schnittpunkte zwischen Grenzertrags- und Grenzkostenkurve bestehen. In diesen Perioden existieren somit zwei lokale Maxima. Hier vergleicht der Manager die lokalen 
Maxima und sucht das globale Maximum heraus. Dies kann zu massiver Gewinndestabilisierung führen. Insbesondere ist dies dann zu erwarten, wenn auch ohne Gewinnmanipulation der Grenzertrag stark im Zeitablauf schwankt (wie bei zahlreichen New-Economy Firmen). Dann ist es für den Manager attraktiv, den bereits niedrigen Grenzertrag in einer Periode noch weiter durch Gewinnmanipulation zu senken (und damit das erste lokale Maximum zu realisieren) und den bereits hohen Grenzertrag in einer anderen Periode noch weiter zu erhöhen (und damit das zweite lokale Maximum zu realisieren). Diese Manipulation würde der Manager in extremer Weise betreiben, gäbe es keine direkten Kosten der Manipulation. Diese beschränken allerdings das Manipulationsausmaß.

Dies sei an einem Beispiel verdeutlicht. Wiehler (2003) betrachtet ein Start-upUnternehmen über drei Perioden. In der ersten Periode wird ein Verlust $k_{1}=-3$ erwartet, in der zweiten ein Gewinn $k_{2}=5$ und in der dritten ein Gewinn $k_{3}=8$. Der Manager erhält ein Fixum $\mathrm{f}=0,5$ pro Periode und eine periodische Erfolgsbeteiligung von $\gamma=10 \%$. Seine Risikoaversion ist $\mathrm{b}=1$, sein Zeitpräferenzfaktor $\beta_{t}=1,1^{-t}$. Weiter gelten die Kostenfaktoren $\quad c_{e}=c_{\Delta}=0,01$. Die Gewinnvolatilität beträgt $\sigma=\sqrt{0,5}$ in allen Perioden.

Die folgende Tabelle zeigt die optimale Gewinnmanipulation und den optimalen Arbeitseinsatz bei verschiedenen Vektoren der Basiskurse $\left(\mathrm{B}_{1}, \mathrm{~B}_{2}, \mathrm{~B}_{3}\right)$. 


\begin{tabular}{|rrrr|}
\hline Basispreis-Vektor & $3 / 3 / 3$ & $0 / 0 / 0$ & $-3 / 5 / 8$ \\
\hline delta 1. Periode & -1 & $-0,8$ & 0,2 \\
\hline Arbeitseinsatz 1. Periode & 0 & 0 & 2,4 \\
delta 2. Periode & 0,7 & 0,6 & 0 \\
\hline Arbeitseinsatz 2. Periode & 2 & 1,6 & 2,5 \\
delta 3. Periode & 0,3 & 0,2 & $-0,2$ \\
\hline Arbeitseinsatz 3. Periode & 1,6 & 1,2 & 2,5 \\
Erwartungsnutzen & $-1,305$ & $-1,139$ & $-1,527$
\end{tabular}

$\square$ Manipulation 1. Periode Manipulation 2. Periode 口Manipulation 3. Periode

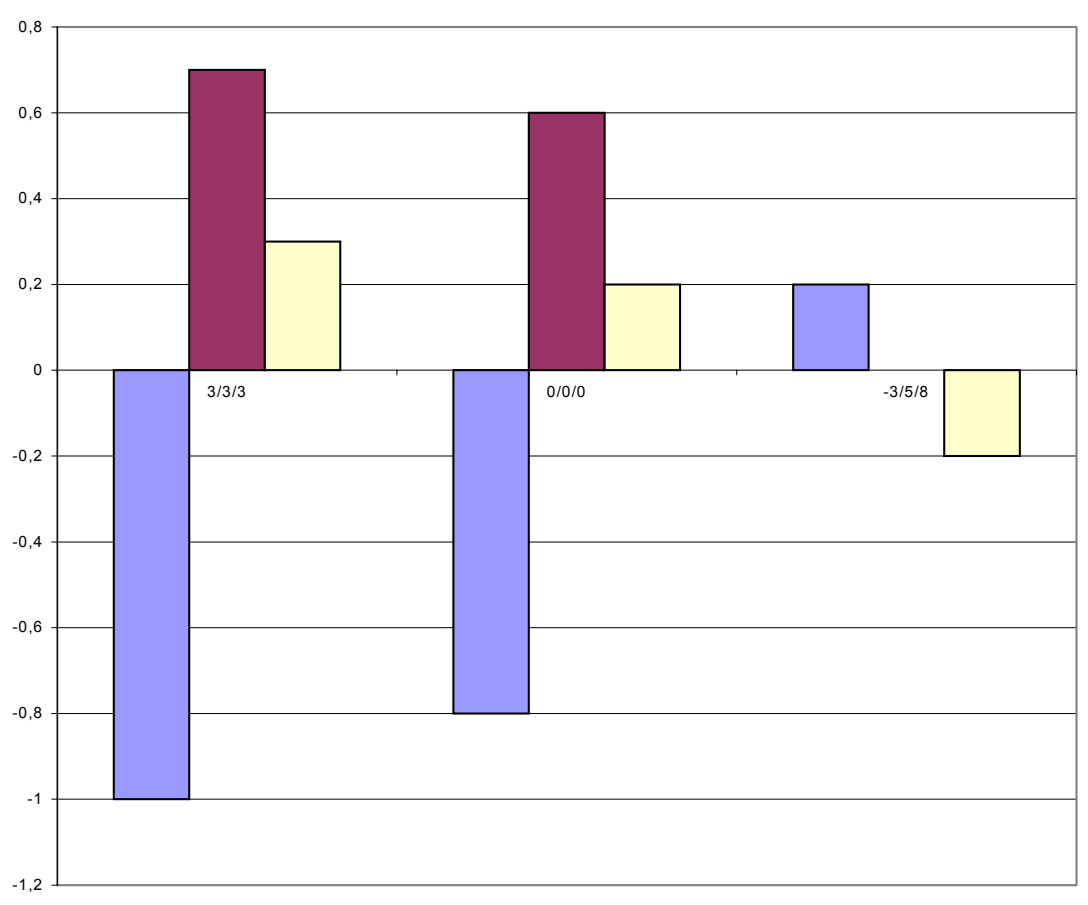

Abb. 3: Gewinnmanipulation und Arbeitseinsatz bei einem Start up-Unternehmen und unterschiedlichen Basiskurs-Vektoren $\left(B_{1}, B_{2}, B_{3}\right)$

Zunächst werde der Basiskurs in jeder Periode auf $k_{2}=3$ festgesetzt. Eine Gewinnbeteiligung ist daher für den Manager in der ersten Periode sehr unwahrscheinlich. Folglich erhöht er den Verlust in der ersten Periode durch Manipulation bei minimalem Arbeitseinsatz. Gleichzeitig erhöht er den Gewinn vor allem in der zweiten Periode, weniger in der dritten, in der die Erfolgswahrscheinlichkeit sowieso hoch ist. Parallel erhöht er seinen Arbeitseinsatz in der zweiten und dritten Periode, vor allem in der zweiten. 
Wird der Basiskurs in allen Perioden auf 0 gesenkt, dann ist die Erfolgswahrscheinlichkeit in jeder Periode höher. In der ersten Periode ist sie jedoch nach wie vor gering. Daher erhöht der Manager wiederum den Verlust in der ersten Periode bei minimalem Arbeitseinsatz. Allerdings fallen die Manipulationen nun in allen drei Perioden geringer aus, weil die Unterschiede in den Erfolgswahrscheinlichkeiten geringer sind. Auch ist der Arbeitseinsatz in der zweiten und dritten Periode geringer. Dies, obwohl die Erfolgswahrscheinlichkeiten höher sind. Die Erklärung liegt im abnehmenden Grenznutzen des Einkommens, das nun mit hoher Wahrscheinlichkeit deutlich höher ist.

Wird der Basiskurs in jeder Periode dem erwarteten Gewinn $k_{t}$ gleichgesetzt, dann entfällt die Manipulation fast vollständig. Gleichzeitig kommt es in jeder Periode zu einem hohen Arbeitseinsatz, da der Grenzertrag der Arbeit stets hoch ist. Diese Konstellation mag den Gesellschaftern besonders wünschenswert erscheinen, bringt allerdings dem Manager einen vergleichsweise niedrigen Erwartungsnutzen. Dies erklärt sich aus den vergleichsweise hohen Basiskursen in der zweiten und dritten Periode.

Die Folge hoher Gewinnmanipulation ist eine hohe „Volatilität“ des Gewinnausweises. Sie erhöht insgesamt die erwartete Entlohnung des Managers. Dieser Effekt kann für die Aktionäre erheblich sein. Eine Senkung des Gewinns in der ersten Periode um 1 Einheit senkt die erwartete Gewinnbeteiligung in dieser Periode um den Betrag $\gamma_{1} N\left(\left(\mu_{1}-B_{1}\right) / \sigma_{1}\right)$ und erhöht sie in der zweiten Periode um $\gamma_{2} N\left(\left(\mu_{2}-B_{2}\right) / \sigma_{2}\right)$. Der Manager kann bei entsprechendem Datenkranz einen substantiellen Vorteil erzielen.

Derartiges Managerverhalten steht im Einklang mit der Optionstheorie. Der Wert einer Option wächst mit der Volatilität des zugrundeliegenden Instruments. Die Gewinnbeteiligung ist eine mehrperiodige Option. Um ihren Wert zu erhöhen, kann es für den Manager zweckmäßig sein, die Volatilität des Gewinnausweises durch Manipulation zu erhöhen. 


\section{Implikationen für die Entlohnungspolitik}

Die Implikationen, die die Gesellschafter eines Unternehmens aus den vorangegangenen Ergebnissen für die Entlohnungspolitik ableiten, hängen von ihrer Zielsetzung ab. Eine mögliche Zielsetzung besteht darin, den Barwert der erwarteten Gewinne nach Abzug der Managervergütung zu maximieren. Hierbei werden Risikoprämien vernachlässigt. Dann wäre die intertemporale Volatilität des Gewinnausweises als solche für die Gesellschafter irrelevant. Ihre Bedeutung beschränkte sich dann auf den Barwert der Managerentlohnung sowie ihre Rückwirkungen auf den Arbeitseinsatz des Managers. Nicht angesprochen werden soll hier die Problematik unterschiedlicher Zeitpräferenzen von Manager und Gesellschaftern (Laux/Liermann 2003, Kap. XXV, 4).

Wenn es zu hoher Volatilität des Gewinnausweises kommt, dann kommt es wie gezeigt auch zu hoher Volatilität des Arbeitseinsatzes des Managers. Er wird sich in den Perioden mehr anstrengen, in denen die Erfolgswahrscheinlichkeit höher ist. Ein hoch volatiler Arbeitseinsatz des Managers kann den Barwert der erwarteten Gewinne beeinträchtigen und so die Gesellschafter treffen. $\mathrm{Ob}$ sie eine hohe Volatilität auch wegen der damit verbundenen höheren Entlohnung ablehnen, ist indessen offen. Wenn sie dies korrekt antizipieren, können sie das Fixum entsprechend der Partizipationsbedingung des Managers anpassen.

Offen bleibt, ob bei hoher Volatilität des Gewinnausweises der Manager insgesamt über alle Perioden hinweg ein höheres Einkommensrisiko tragen muss. In dem geschilderten, sehr vereinfachten Modell kann die Volatilität der Gewinnstörungen vom Manager nicht beeinflusst werden. Andernfalls ist sehr wohl vorstellbar, dass er eine riskantere Unternehmenspolitik betreiben wird, soweit dies den Wert seiner Gewinnbeteiligung erhöht ${ }^{4}$.

Sind die Gesellschafter ebenfalls risikoscheu, dann werden sie durch eine höhere Verhaltensvolatilität benachteiligt. Dies ist ein weiterer Grund, eine Entlohnungspolitik zu betreiben, die den Manager nicht zu hoher Verhaltensvolatilität motiviert, abgesehen von Vertrauensproblemen, die damit früher oder später einhergehen können. Von dieser

4 Dies erklärt, weshalb es in den USA verboten ist, den Managern von öffentlich vertriebenen Investmentfonds eine optionsähnliche erfolgsabhängige Vergütung zu zahlen. 
Aussage $\mathrm{zu}$ trennen ist die Frage, ob die Gesellschafter eine Bilanzpolitik der Gewinnglättung wünschen oder nicht. Durchschauen sie jede Gewinnglättung, dann sollte sie ohne Wirkung bleiben. Andernfalls lassen sich Argumente pro und contra anführen.

Wie sieht eine Entlohnungspolitik aus, die die Verhaltensvolatilität einschränkt? Im o.a. Beispiel genügte es, in jeder Periode den Basiskurs $B_{t}=k_{t}$ zu setzen, um Gewinnmanipulationen weitgehend auszuschalten. Gemäß Gleichung (7) ist $\Delta_{t}=0$, wenn $\beta_{t} *$ Grenzertrag ohne Manipulation in allen Perioden gleich hoch ist. Dies kann gemäß Gleichung (5) angestrebt werden, indem der Basiskurs und das Fixum entsprechend variiert werden. Sind $\beta_{t}, \gamma_{t}$ und $\sigma_{t}$ in allen Perioden gleich hoch, dann genügt es, die Differenz zwischen erwartetem Gewinn $k_{t}$ und Basiskurs $B_{t}$ sowie das Fixum $f_{t}$ über die Zeit konstant zu halten. Eine Gewinnmanipulation ist dann für den Manager unattraktiv.

Ist von vornherein absehbar, in welchen Perioden mit hohen (niedrigen) Gewinnen vor Manipulation zu rechnen ist, dann sollten die Basiskurse entsprechend periodenbezogen gewählt werden. Hierbei können außerdem periodenabhänge Werte von $\beta$ und $\sigma$ berücksichtigt werden.

Ein anderer Parameter der Entlohnungspolitik ist die im Modell nicht explizit betrachtete Wahl der Periodenlänge. Wird die Periodenlänge z.B. verdoppelt, dann gleichen sich Gewinnmanipulationen zum Teil automatisch innerhalb dieses Zeitraumes aus. ${ }^{5}$ Die Wirkung von Gewinnmanipulationen wird so gemindert. Zweitens kommt es zu intertemporaler Risikostreuung. Das $\sigma_{t}$ über zwei Perioden ist lediglich gleich der Wurzel aus der Summe der beiden quadrierten Perioden- $\sigma$, sofern die Gewinnstörungen beider Perioden unabhängig voneinander sind. Das $\mu$ über beide Perioden ist jedoch gleich der Summe der Perioden- $\mu$. Eine Verlängerung der Periode reduziert somit den Einfluss des Risikos. Dies reduziert auch die Volatilität der Erfolgswahrscheinlichkeit.

5 Dann erscheint auch die Modellprämisse, wonach der Manager über die Manipulation vor Bekanntwerden der Gewinnstörung entscheidet, weniger problematisch. 
Ein weiterer Parameter der Entlohnungspolitik ist die Beteiligungsquote $\gamma_{t}$. Wie aus der ersten Zeile von Gleichung (3) ersichtlich, steigt der Grenzertrag $\partial U_{t} / \partial \mu_{t}$ nicht zwingend mit $\gamma_{t}$. Das liegt daran, dass mit steigendem $\gamma_{t}$ der Grenznutzen des Einkommens abnimmt. Jedoch ist dies ein Effekt nachgelagerter Größenordnung im Vergleich zu dem unmittelbaren Einkommenseffekt einer Erhöhung von $\gamma_{t}$. Zudem wird er eingeschränkt, wenn das Fixum bei Erhöhung der Beteiligungsquote gesenkt wird. Daher kann man bei realistischen Werten davon ausgehen, dass der Grenzertrag mit $\gamma_{t}$ wächst.

Sollen in einer Periode mit niedrigen Gewinnerwartungen zusätzliche Leistungsanreize geboten werden, so kommt dafür auch eine Erhöhung von $\gamma_{t}$ in Betracht, anstelle von oder in Ergänzung zu einer Senkung des Basiskurses. Dies ist insbesondere dann der Fall, wenn die Erfolgswahrscheinlichkeit bereits hoch ist und diesbezüglich kein Handlungsbedarf besteht. Auch so kann die Verhaltensvolatilität eingeschränkt werden.

\section{Implikationen für Aktienoptionspläne und Aktienpläne}

In den letzten Jahren haben auch in Deutschland Aktienoptionspläne zunehmend Gewinnbeteiligungssysteme abgelöst. Inwieweit lassen sich die vorangehenden Erkenntnisse auf Aktienoptionspläne übertragen? Laux 1990, 1991 hat darauf hingewiesen, dass der mit solchen Plänen beabsichtigte Leistungsanreiz nur dann entstehen kann, wenn die Manager die Risiken aus solchen Plänen nicht durch private Transaktionen hedgen können. Das gilt auch bei anderen erfolgsabhängigen Vergütungssystemen.

Bei Aktienoptionsplänen spielt die Kursentwicklung der Aktien des Unternehmens die entscheidende Rolle für die Gewinne des Managers aus der Optionsausübung. Auch wenn der Kurs von zahlreichen Faktoren getrieben wird, so spielt doch der Gewinnausweis eine wichtige Rolle. Das zeigt sich daran, dass es für viele Unternehmen ein Kurs-Gewinn-Verhältnis gibt, das als Richtschnur für eine angemessene Bewertung der Aktien angesehen wird. Gelingt es dem Manager, durch eine Gewinnmanipulation den Gewinn pro Aktie um 1 Euro zu erhöhen, ohne dass dies 
als Manipulation erkannt wird, dann steigt der Aktienkurs etwa um 20 Euro, ausgehend von einem Kurs-Gewinn-Verhältnis von 20. Wenn der Manager einen Bruchteil $\gamma(=$ Zahl von Kaufoptionen durch Zahl der ausstehenden Aktien des Unternehmens) hält und diese Optionen im Geld sind, dann gewinnt der Manager durch die Gewinnmanipulation den Betrag „Zahl der Optionen * 20“, also „ $\gamma *$ Zahl der ausstehenden Aktien*20“. Damit hält er einen gewichtigen Bereicherungshebel in Händen. Entsprechend hoch ist sein Anreiz zur Gewinnmanipulation. Dies gilt auch, wenn die Ausübungsfrist erst nach Ablauf einiger Jahre beginnt. Eine solche Frist wirkt ähnlich der bereits angesprochenen Verlängerung der Periodenlänge bei Gewinnbeteiligung. Aus dem Vorangehenden folgt, dass der Anteil $\gamma$ bei Aktienoptionsplänen sehr viel niedriger als bei Gewinnbeteiligungsplänen gewählt werden sollte. Andernfalls werden die Bilanzmanipulationen, die in jüngster Zeit gemeldet wurden, zahlreiche Nachahmer finden.

Häufig wird in Deutschland bei Aktienoptionsplänen ein Basiskurs gewählt, der deutlich über dem Aktienkurs bei Ausgabe der Optionen liegt. ${ }^{6}$ Hiermit soll ein Anreiz zu hoher Leistung der Manager geschaffen werden. Die vorangehenden Überlegungen zeigen, dass diese Vorgehensweise auch Gefahren birgt. Erstens ist die Ausübungswahrscheinlichkeit dadurch relativ niedrig. Eine Gewinnmanipulation mag dann bei schwacher Gewinnentwicklung das einzige Mittel sein, die Optionen ins Geld zu bringen. Zweitens sinkt der Wert der Optionen bei sinkendem Aktienkurs, weil die Ausübungswahrscheinlichkeit sinkt. Insbesondere tendiert das Optionsdelta gegen 0 . Der Leistungsanreiz wird dadurch abgeschwächt. Die Manager reagieren darauf oft mit der Forderung, den Basiskurs nachträglich zu senken oder neue Optionen auszugeben. Sie argumentieren, es gäbe sonst nicht genügend Leistungsanreize. Dieses Argument mag zwar stimmen. Die nachträgliche Aufwertung der Optionen transformiert jedoch die ursprüngliche Optionsvergütung in eine weitgehend kursunabhängige Vergütung. Wird dies zur Regel, dann wird der beabsichtigte Leistungsanreiz weitgehend beseitigt.

Dieses Nachverhandlungsproblem wird gemildert, wenn die Manager statt Optionen Aktien erhalten. Dann sinkt zwar bei schlechten Unternehmensergebnissen der

6 Gemäß $§ 193$, Abs. 2 (4) AktG müssen bei Aktienoptionsprogrammen auch Erfolgsziele festgelegt werden. 
Aktienkurs, jedoch bleibt das Kurssteigerungspotential erhalten, denn das Delta der Aktie ist stets gleich 1. Die Aktie ist sozusagen immer im Geld. Damit bleibt der Leistungsanreiz erhalten. Aber auch bei Aktienentlohnung besteht wie bei Optionen der erhebliche Bereicherungshebel des Kurs-Gewinn-Verhältnisses. Daher ist auch hier entsprechende Vorsicht bei der Wahl des Anteils $\gamma$ geboten, also der Zahl der an den Manager ausgegebenen Aktien relativ zur Zahl der ausstehenden Aktien.

\section{Fazit}

Der Glaube an die Informationseffizienz des Kapitalmarktes hat in jüngster Zeit stark gelitten. Glaubten viele, die Akteure des Kapitalmarktes ließen sich durch Bilanzmanipulationen nicht in die Irre führen, so sehen sie sich getäuscht. Der Jahresabschluß ist nach wie vor eine der wichtigsten Informationsquellen für den Kapitalmarkt. Die Messung des Gewinns ist indessen infolge der notwendigerweise in sie einfließenden Zukunftserwartungen des Bilanzierenden ein Unterfangen, das zahlreichen subjektiven Einflüssen unterliegt. Daher sind Manipulationen häufig von außen nicht erkennbar. Manager besitzen einen erheblichen Spielraum , mit dem sie den Gewinn und damit auch den Aktienkurs manipulieren können.

Daraus folgt, dass gewinnabhängige und aktienkursabhängige Entlohnungssysteme nicht nur unter dem Aspekt von Leistungsanreiz und Risikoprämien konzipiert werden dürfen, sondern auch die dadurch entstehenden Anreize zur Manipulation berücksichtigt werden müssen. Für den Manager, der viele Aufgaben erfüllen muss, stellt sich die Frage, wie viel Zeit er in wirkungsvolles realwirtschaftliches Management und wie viel Zeit er in Bilanzpolitik investieren soll. Je schwieriger es für ihn ist, durch effektives realwirtschaftliches Management Erfolge zu erzielen, desto attraktiver ist es, mehr Kraft in die Bilanzpolitik zu investieren. Dies ermöglicht ihm am ehesten, sein Einkommen zu erhöhen (zu Lasten der Aktionäre).

$\mathrm{Ob}$ es gelingt, durch zusätzliche Kontrollmechanismen und Sanktionsdrohungen die Kapitalmarktverfassung so weit zu verbessern, dass die Manipulationsgefahr deutlich vermindert wird, darf bezweifelt werden. Der Gewinn, zu dessen Erzielung der Manager beiträgt, ist eben auch bei ausgefeiltesten Regeln der Rechnungslegung nicht so präzise zu ermitteln wie die Zahl der tadellosen Werkstücke, die ein Arbeiter im Akkord fertigt. Dementsprechend kann der Manager nicht „im Akkord“ entlohnt werden. So bleiben die 
Möglichkeiten der gewinnabhängigen Managerentlohnung beschränkt. Vielleicht ist dies ein Anlaß für marktorientierte Gesellschaftssysteme, nicht nur auf extrinsische finanzielle Anreize für Manager zu setzen, sondern auch die intrinsischen Anreize durch Erziehung und Bildung zu verstärken. Managern, deren Leistungsmotivation nur auf extrinsischen Anreizen beruht, werden die Gesellschafter von vornherein mit Skepsis begegnen. Eine anspruchsvolle Aufgabe, gepaart mit Verantwortung für das Wohlergehen zahlreicher Stakeholder, sollte Ansporn für Leistung sein. 
Anhang: Ableitung von Gleichung (3)

Es genügt,

$$
H_{t} \equiv \int_{B_{t}}^{\infty} \exp \left(-b \gamma_{t}\left(g_{t}-B_{t}\right)\right) d N\left(g_{t} ; \mu_{t}, \sigma_{t}\right)
$$

zu betrachten.

$$
\begin{aligned}
H_{t} & =\int_{B_{t}}^{\infty} \exp \left(-b \gamma_{t}\left(g_{t}-B_{t}\right)\right) \frac{1}{\sigma_{t} \sqrt{2 \pi}} \exp \left(-\frac{1}{2}\left(\frac{g_{t}-\mu_{t}}{\sigma_{t}}\right)^{2}\right) d g_{t} \\
& =\exp \left(b \gamma_{t} B_{t}\right) \int_{B_{t}}^{\infty} \frac{1}{\sigma_{t} \sqrt{2 \pi}} \exp \left(-\frac{1}{2}\left(\frac{g_{t}-\left(\mu_{t}-b \gamma_{t} \sigma_{t}^{2}\right)}{\sigma_{t}}\right)^{2}\right) d g_{t} \exp \left(-\frac{1}{2}\left(\frac{\mu_{t}}{\sigma_{t}}\right)^{2}+\frac{1}{2}\left(\frac{\mu_{t}-b \gamma_{t} \sigma_{t}^{2}}{\sigma_{t}}\right)^{2}\right) \\
& =\exp \left(b \gamma_{t} B_{t}\right) N\left(\frac{\mu_{t}-b \gamma_{t} \sigma_{t}^{2}-B_{t}}{\sigma_{t}}\right) \exp \left(\frac{1}{2}\left(b \gamma_{t} \sigma_{t}\right)^{2}-b \gamma_{t} \mu_{t}\right) \\
& =\exp \left(\frac{1}{2}\left(b \gamma_{t} \sigma_{t}\right)^{2}\right) \exp \left(-b \gamma_{t}\left[\mu_{t}-B_{t}\right]\right) N\left(v_{t}\right) \\
& =\exp \left(-\frac{1}{2}\left(b \gamma_{t} \sigma_{t}\right)^{2}\right) \exp \left(-b \gamma_{t} \sigma_{t}\left[\frac{\mu_{t}-B_{t}-b \gamma_{t} \sigma_{t}^{2}}{\sigma_{t}}\right]\right) N\left(v_{t}\right) \\
& =\exp \left(-\frac{1}{2}\left(b \gamma_{t} \sigma_{t}\right)^{2}\right) \exp \left(-b \gamma_{t} \sigma_{t} v_{t}\right) N\left(v_{t}\right)
\end{aligned}
$$

Hieraus ergibt sich Gleichung (3). 


\section{Literaturverzeichnis}

Carpenter, Jennifer N. (2000): Does Option Compensation Increase Managerial Risk Appetite? Journal of Finance, vol. 55, S. 2311-2331.

Claussen, Carsten Peter; Bröcker, Norbert (2002): Der Corporate Governance-Kodex aus der Perspektive der kleinen und mittleren Börsen-AG. Der Betrieb, S. 1199-1206.

Das, Sanjiv Ranjan; Sundaran, Rangarajan K. (2002), Fee Speech: Signaling, RiskSharing and the Impact of Fee Structures on Investor Welfare. Review of Financial Studies, vol. 15, S. 1465-1497.

Degeorge, François; Patel, Jayendu; Zeckhauser, Richard (2002): Earnings Management to Exceed Thresholds. Forthcoming in: Journal of Business.

Franke, Günter; Hax, Herbert (1999): Finanzwirtschaft des Unternehmens und Kapitalmarkt. 4. Aufl., Berlin et al.

Gao, Pengjie, Shrieves, Ronald E. (2002): Earnings Management and Executive Compensation: a Case of Overdose of Option and Underdose of Salary? Working paper, University of Tennessee.

Gillenkirch, Robert (1997): Gestaltung optimaler Anreizverträge. Wiesbaden.

Laux, Helmut (1990): Die Irrelevanz erfolgsorientierter Anreizsysteme bei bestimmten Kapitalmarktbedingungen - Der Einperiodenfall. Zeitschrift für Betriebswirtschaft 60 . Jg., S. 1341-1358.

Laux, Helmut (1991): Die Irrelevanz erfolgsorientierter Anreizsysteme bei bestimmten Kapitalmarktbedingungen - Der Mehrperiodenfall. Zeitschrift für Betriebswirtschaft 61 . Jg., S. 477-488.

Laux, Helmut (1999): Unternehmensrechnung, Anreiz und Kontrolle. 2. Auflage, Berlin.

Laux, Helmut (2003): Wertorientierte Unternehmensführung und Kapitalmarkt, Berlin et al. 
Laux, Helmut; Liermann, Felix (2003): Grundlagen der Organisation. 5. Auflage, Berlin.

Murphy, Kevin J. (1999): Executive Compensation. In: Handbook of Labor Economics, hrsg. von O. Ashenfelter, D. Card, Amsterdam et al., S. 2485-2563.

Myers, Stewart C. (1977): Determinants of Corporate Borrowing. Journal of Financial Economics vol. 5, S. 147-175.

Spremann, Klaus (1987): Agent and Principal. In Agency Theory, Information, and Incentives, hrsg. v. G. Bamberg, K. Spremann, Berlin, S. 3-37.

Wiehler, Stephan (2003): Managerentlohnung und Bilanzpolitik. Diplomarbeit Universität Konstanz. 